\title{
DESTILAÇÃO DA MiSCELA II: MODELAGEM E SIMULAÇÃO DO STRIPPING DO HEXANO ${ }^{1}$
}

\author{
Paulo R. PARAÍSO ${ }^{2, *}$, Cid M. G. ANDRADE², Roger J. ZEMP
}

\begin{abstract}
RESUMO
No processo de extração do óleo de soja forma-se a Miscela, que é uma mistura de óleo e hexano. O processo de separação destes dois componentes é denominado de Destilação da Miscela. Esta é composta basicamente da operação de evaporação e da operação de stripping do hexano. Neste trabalho, desenvolveu-se uma modelagem matemática para o stripping do hexano. O modelo é baseado em balanços de massa e energia e em relações de equilibrio; e, a sua validação foi feita comparando-se os resultados obtidos com os dados operacionais da indústria da COAMO (Cooperativa Agrícola Mouraoense). Em seguida, com o modelo elaborado, fez-se as simulações para estudar: as influências da concentração da alimentação e do modo de injeção de vapor direto nos resultados operacionais do stripper.

Palavras-chave: modelagem; simulação; miscela; stripping.
\end{abstract}

\section{SUMMARY}

DISTILLATION OF THE MISCELLA II: MODELING AND SIMULATION OF THE HEXANE STRIPPING. In the soybean oil extraction process make the Miscella that is a mixture of oil and hexane. The separation process in both components is named Miscella Distillation. It is composed mainly of the hexane evaporation and of the hexane stripping. In this work, it was developed a mathematical modeling of the hexane stripping. This model is based in mass and energy balances and in equilibrium relations. The validity of the model was made comparing the obtained results with data from a industry of the COAMO (Cooperativa Agricola Mouraoense). In following, with the elaborated model, it was made the simulation of the stripping to study: the influences of the feed flow and the injection form of direct steam in the operation results of the stripper.

Keywords: modeling; simulation; miscella; stripping.

\section{1 - INTRODUÇÃO}

A industrialização de oleaginosas constitui-se num importante setor do sistema agroindustrial, pela importância de seus produtos nas indústrias siderúrgicas, de cosméticos e como matéria-prima na indústia de alimentos para o consumo animal e humano, segundo BARBOSA FREITAS \& FRANÇA [1]. Os produtos gerados atendem o mercado interno e externo, e para serem competitivos, necessitam sempre buscar a redução de custos.

Uma estratégia visando a redução de custos, bem como a proteção do meio ambiente e do consumidor, nas indústrias já instaladas, é procurar sempre estabelecer as melhores condições de operação do processo. Tais condições podem propiciar um consumo menor de energia e um nível mais elevado de recuperação de hexano que podem ser importantes tanto na redução de custos quanto na proteção do meio ambiente e do consumidor. As indústrias instaladas no Paraná/Brasil utilizam o processo contínuo de extração por solvente, sendo o solvente utilizado o hexano. As operações do processo que deverão ser as principais numa estratégia de redução de custos operacionais são: a extração do óleo por solvente, a destilação da miscela e a dessolventização-

\footnotetext{
1. Recebido para publicação em 28/06/2002. Aceito para publicação em 28/12/2004 (000915).

2. Departamento de Engenharia Quimica, UEM-Maringá. E-mail: paulo@deq.uem.br,cid@deq.uem.br

3. Departamento de Sistemas Quimicos-FEQ/UNICAMP-Campinas. E-mail: zemp@desq.feq.unicamp.br

* A quem a correspondência deve ser enviada.
}

tostagem do farelo uma vez que estas consomem muita energia e a manipulam com o hexano.

A miscela é uma mistura entre óleo de soja e o hexano formada no extrator. A separação destes componentes é denominada de Destilação da Miscela, que se compõe: da evaporação e do stripping do hexano. Nestas operações, quantidades expressivas de energia são utilizadas, na forma de vapor.

Este trabalho apresenta a modelagem matemática e simulação da etapa de evaporação do hexano. A validação do modelo desenvolvido sera feito através da comparação dos dados gerados com os dados operacionais da indústria da COAMO (Cooperativa Agrícola Mouraoense). Na seqüência, um conjunto de simulações serão executadas a fim de estudar: influência da concentração da alimentação e do modo de injeção de vapor direto nos resultados operacionais do stripper.

\section{2 - MATERIAL E MÉTODOS}

\section{1 - O stripper}

Uma etapa de extrema importância na destilação da miscela é a operação de stripping do óleo. Esta tem a função de finalizar a destilação da miscela, ou seja, deve haver a separação do baixo teor de hexano que ainda permanece no óleo após a operação de evaporação. O equipamento utilizado neste tipo de operação é denominado de stripper e é mostrado no esquema da Figura 1.

A Figura 1 mostra o esquema de um stripper típico utilizado numa planta de obtenção de óleo bruto de soja, que é uma coluna dividida em compartimentos denomi- 
nados de estágios e com estruturas específicas para propiciar o fluxo e o contato dos materiais. A miscela concentrada em óleo forma a corrente liquida que entra na parte superior e passa por uma estrutura que facilita sua distribuição dentro do equipamento. Esta corrente ao atingir o estágio forma um pequeno reservatório de líquido, que possibilita o contato entre o vapor direto e a miscela. Este vapor entra no fundo do estágio, que é o distribuir no seio do líquido de modo a ocorrer o stripping do hexano presente no óleo. Após o reservatório atingir um certo nível, o líquido flui ao estágio inferior através de uma tubulação instalada externamente, conforme mostra a Figura 1. Este fluxo da corrente líquida ocorre até que o óleo bruto saia no fundo do stripper. A corrente de vapor, composta de vapor d'água e hexano, flui de maneira contracorrente em relação à corrente líquida e passa de um estágio inferior para o superior através de tubulações também instaladas externamente, conforme mostra a Figura 1. Assim, pode-se observar que o fluxo de correntes, de estágio a estágio, neste equipamento se diferencia um pouco da forma tradicional. Ele se diferencia na medida em que a corrente de vapor que sobe e a corrente de líquido que desce não se cruzam no fundo do estágio uma vez que este é fechado, como mostra o esquema da Figura 1.

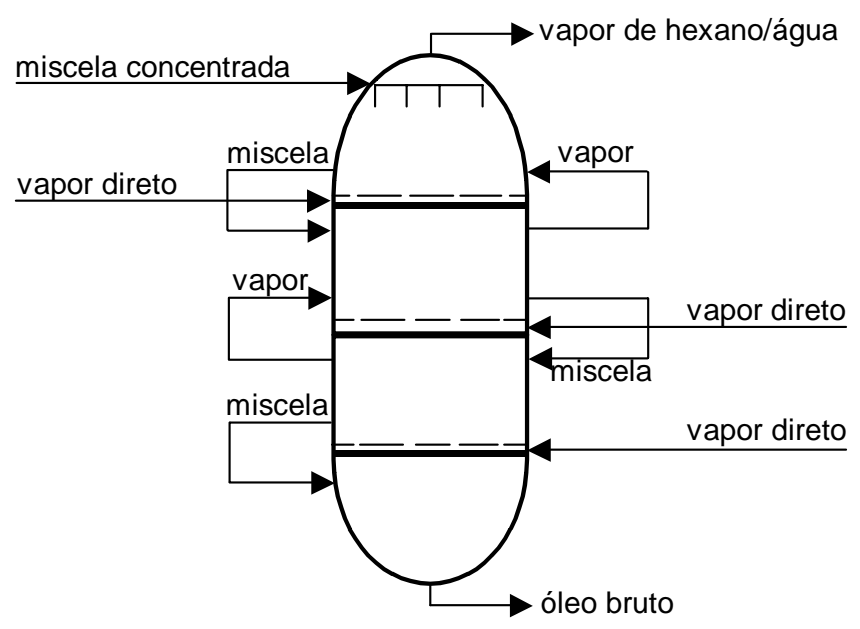

FIGURA1. Esquema do stripper

O stripper é um equipamento bem específico e para maximizar a recuperação do hexano, KEMPER [6] recomenda que a miscela na entrada tenha uma concentração em massa de óleo de $97 \%$ ou mais e uma temperatura superior a $107^{\circ} \mathrm{C}$; o vapor deve ser superaquecido com temperatura de $177^{\circ} \mathrm{C}$ e uma pressão em torno de $4 \mathrm{kgf} / \mathrm{cm}^{2}$.

O óleo destilado no stripper deve ser testado a fim de verificar se ainda contém traços de hexano. O teste é feito pelo "ponto de fulgor", que é definido como o ponto mais baixo da temperatura em que os vapores saídos do líquido, misturados com o ar, e em contato com uma chama, queimam na superficie desse líquido. A Tabela 1 mostra o ponto de fulgor $\left({ }^{\circ} \mathrm{C}\right)$ do hexano no óleo de soja e a sua respectiva concentração (ppm).
TABELA 1. Ponto de fulgor versus hexano residual.

\begin{tabular}{cc}
\hline ponto de fulgor $\left({ }^{\circ} \mathrm{C}\right)$ & hexano residual $(\mathrm{ppm})$ \\
\hline 82 & 3500 \\
99 & 1600 \\
121 & 1000 \\
160 & 550 \\
\hline
\end{tabular}

\section{2 - Modelo matemático do stripper}

A Figura 2 mostra o esquema do stripper que se pretende modelar. Este se apresenta como uma coluna dividida em estágios onde o vapor entra em contato direto e em contracorrente com a miscela concentrada, arrastando, assim, o hexano e formando uma corrente de gases que sai no topo; no fundo sai a corrente de óleo bruto praticamente livre do hexano.

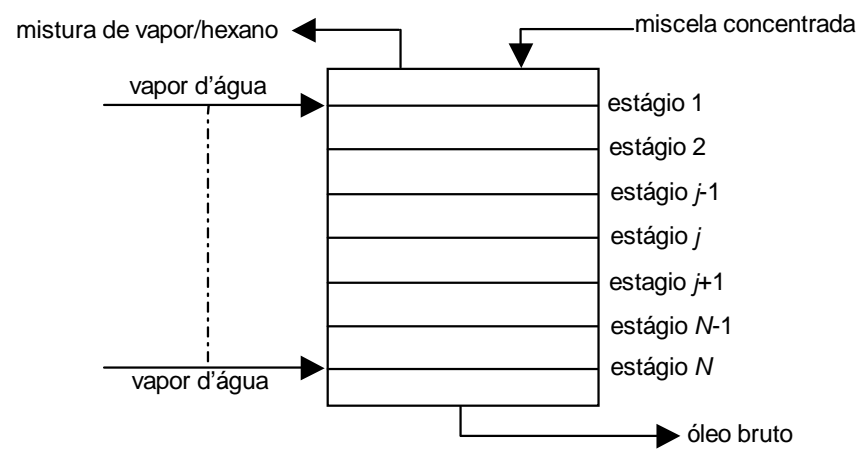

FIGURA 2. Esquema geral do stripper na separação óleo de soja/hexano

Os modelos matemáticos que representam o stripper são baseados em balanços de massa e de energia, equações de equilíbrio e relações empíricas.

Existem na literatura, vários algorítmos para a solução do sistema de equações resultante da modelagem prato a prato de processos de separação. Neste trabalho, pretende-se utilizar o algoritmo do método SR (SumRates) de Burningham-Otto, segundo HENLEY \& SEADER [2]. A escolha se justifica uma vez que ele é um método de solução rigoroso e se adequa muito bem ao sistema, pois existe uma diferença significativa de volatilidade entre os componentes do sistema proposto.

O stripper é dividido em estágios e opera continuamente com as correntes de líquido e vapor fluindo, no seu interior, de forma contracorrente. As seguintes hipóteses são assumidas: I) contato entre a fase líquida e a fase de vapor dentro da coluna é perfeito; II) o vapor que sai de cada prato está em equilíbrio com o líquido de saída; III) o vapor d'água é considerado o inerte no processo; IV) o vapor d'água usado dentro da coluna não se condensa; e V) não existem perdas de calor para o meio ambiente.

A modelagem matemática que será utilizada na análise desse extrator é baseada na modelagem geral teórica para separações, em estágio de equilíbrio, já 
apresentada na literatura por vários autores HOLLAND [4], HENLEY \& SEADER [2]. Evidentemente, que no decorrer do desenvolvimento do modelo matemático específico para o caso em estudo serão necessárias algumas adaptações e elas serão feitas.

O esquema do estágio de equilíbrio $j$ é mostrado na Figura 3.

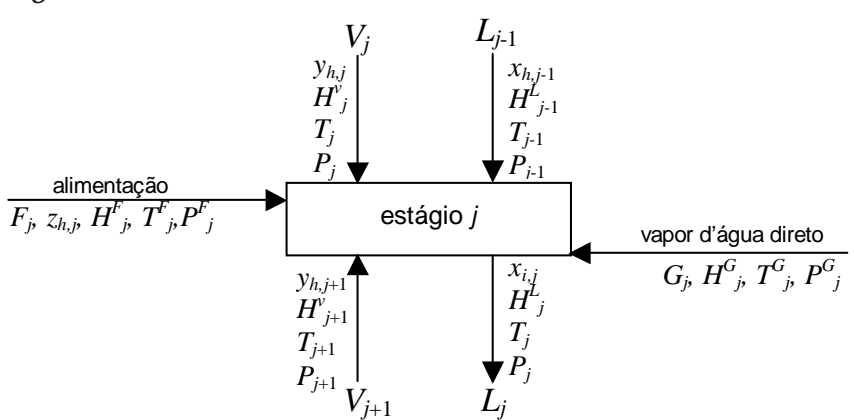

FIGURA 3. Esquema do estágio de equilíbrio $j$

Como se observa pela Figura 3, entrando no estágio $j$ tem-se quatro correntes especificadas de corrente $F_{j}$, de corrente $L_{j-1}$, de corrente $V_{j+1}$ e de corrente $G_{j}$. A corrente $F_{j}$ é a vazão molar de alimentação da mistura com fração molar $z_{h, j}$ do hexano, com temperatura $T^{F}$, com pressão $P_{j}^{F}$ e a correspondente entalpia molar $H_{j}^{F}$ a pressão de alimentação é assumida ser maior ou igual à pressão do estágio $P_{j}$. A corrente $L_{j-1}$ é a vazão molar do líquido que sai do estágio situado acima do estágio $j$ com fração molar $x_{h, j-1}$ do hexano, com temperatura $T_{j-1}$, com pressão $P_{j-1}$ e a correspondente entalpia molar $H_{j-1}$. Assumido-se que a pressão $P_{j-1}$ é menor ou igual à pressão do estágio $j$. A corrente $V_{j+1}$ é a vazão molar dos gases que saem do estágio situado imediatamente abaixo do estágio $j$ com fração molar $y_{h, j+1}$, com temperatura $T_{j+1}$, com pressão $P_{j+1}$ e com a correspondente entalpia $H^{v}{ }_{j+1}$ A corrente $G_{j}$ é a vazão do vapor d'água injetado direto no estágio, com temperatura $T^{G}{ }_{j}$ e com pressão $P_{j}^{G}$. Saindo do estágio $j$ têm-se duas correntes especificadas como corrente $L_{j}$ e como corrente $V_{j}$. A corrente $L_{j}$ é a vazão molar de líquido com composição $x_{h, j}$, com temperatura $T_{j}$, com pressão $P_{j}$ e com a correspondente entalpia molar $H_{j}^{L}$; a corrente $V_{j}$ é a vazão molar do gás com fração molar $y_{h, j}$ de hexano, com temperatura $T_{j}$, com entalpia molar $H^{v}$ e pressão $P_{j}$ a qual está em equilíbrio com a pressão do líquido.

As equações que relacionam todas as variáveis associadas com o estágio teórico $j$ são explicitadas a seguir.

Equação do balanço de massa para o hexano por estágio:

$L_{j-1} x_{h, j-1}+V_{j+1} y_{h, j+1}+F_{j} z_{h, j}-L_{j} x_{h, j}-V_{j} y_{h, j}=0$

Equação do balanço de energia por estágio:

$L_{j-1} H^{L}{ }_{j-1}+V_{j+1} H^{V}{ }_{j+1}+F_{j} H^{F}{ }_{j}+G j H^{G}{ }_{j}-L_{j} H^{L}{ }_{j}-V_{j} H_{j}^{V}=O$

Equação de equilíbrio para o hexano por estágio:

$y_{h, j}=K_{h, j} x_{h, j}$

sendo $K_{h, j}$ a constante de equilíbrio.
O somatório das frações molares dos componentes em cada estágio

$\sum_{i=1}^{c} y_{i, j}=1$

$\sum_{i=1}^{c} x_{i, j}=1$

sendo $c$ o número de componentes da mistura.

A equação do balanço material total pode ser usada no lugar das equações (2.4) e (2.5). Ela pode ser resultante da combinação destas duas equações e a $\sum_{i=1}^{c} z_{i, j}=1$ com a equação (2.1), somando-se para todos c componentes a partir do estágio 1 até o estágio $j$. Assim,

$L_{j}=V_{j+1}+\sum_{m=1}^{j} F_{m}+G_{m}-V_{1}$

A Figura 4 esquematiza um modelo físico de $N$ estágios de uma configuração semelhante ao stripper, a fim de aplicar das equações dos balanços para se obter uma solução para o problema da separação do hexano do óleo de soja, conforme o algoritmo de Burningham-Otto.

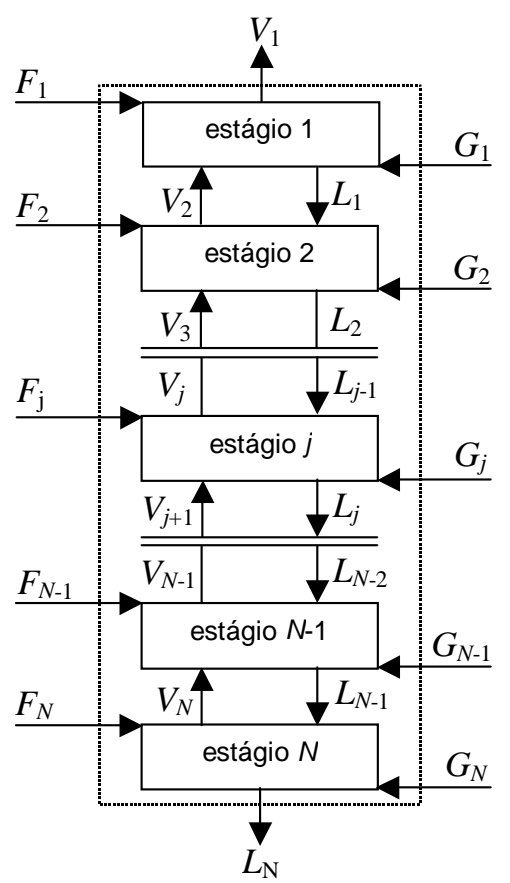

FIGURA 4. Esquema de um extrator contracorrente de $N$ estágios

Uma coluna de $N$ estágios, contracorrente, como mostrado na Figura 4 é representada matematicamente por $N(2 c+3)$ equações, as quais relacionam $N(3 c+9)$ variáveis. Se $N$ e todos $F_{j}, z_{h, j}, T_{j}^{F}, P_{j}^{F}, P_{j}, G_{j}, T_{j}^{G}, P^{G} j$ são especificados, o modelo é representado por $N(2 c+1)$ equações algébricas simultâneas com $N(2 c+1)$ incógnitas, as quais são todos $x_{h, j}, y_{h, j}, L_{j}, V_{j}$ e $T_{j}$ onde as equações do balanço de massa, de energia e as equações de 
equilíbrio são equações não lineares. Evidentemente, outras variáveis podem ser especificadas substituindo as incógnitas mencionadas acima. Independentemente das variáveis especificadas, forma-se um conjunto de equações não lineares que deve ser resolvido mediante técnicas de caráter iterativo.

Existe na literatura diversos métodos que podem ser empregados na solução de balanços de massa por componente, relações de equilíbrio e balanços de energia que ocorrem na modelagem extratores tipo stripper. Em geral, estes procedimentos fazem uso do particionamento de equações em conjunto com a equação de iteração e/ou técnicas de linearização de NewtonRaphson. Entretanto, a escolha do método depende da diferença de volatilidade entre os componentes presentes na mistura a ser separada. Então, uma vez que a volatilidade entre os componentes da mistura em estudo é grande, opta-se pela utilização do método SR (Sum-Rates) para a análise do stripper, em estudo.

Este método foi proposto inicialmente por SUJATA [9] e melhorado por Burningham e Otto, segundo HENLEY \& SEADER [2] mediante a introdução no método do algoritmo da matriz tridiagonal. Esta resulta da forma modificada das equações (2.1) e (2.2) quando estas são transformadas em outras equações utilizando-se de $T_{j}$ e $V_{j}$ como variáveis de iteração. As equações modificadas são lineares em relação às frações molares das correntes liquidas. Este conjunto de equações pode ser resolvido pelo algoritmo de Thomas, segundo HENLEY \& SEADER [2].

A obtenção das equações modificadas resulta da substituição da equação (2.2) na equação (2.1) com o objetivo de eliminar $y$, assim como substituindo a equação (2.6) na equação (2.1) para eliminar $L$. O resultado destas substituições é o conjunto de equações para o hexano e para cada estágio como está escrito a seguir.

$A_{j} x_{h, j-1}+B_{j} x_{h, j}+C_{j} x_{h, j+1}=D_{j}$

sendo:

$$
\begin{aligned}
& A_{j}=V_{j}+\sum_{m=1}^{j-1}\left(F_{m}+G_{m}\right)-V_{1} \quad \text { com } 2 \leq j \leq N \\
& B_{j}=-\left[V_{j+1}+\sum_{m=1}^{j}\left(F_{m}+G_{m}\right)-V_{1}+K_{h, j} \cdot V_{j}\right] \quad \text { com } l \leq j \leq N \\
& C_{j}=K_{h, j+1} V_{j+1} \quad \text { com } \quad l \leq j \leq N-1 \\
& D_{j}=-F_{j} \cdot z_{h, j} \text { com } l \leq j \leq N
\end{aligned}
$$

com $x_{i, 0}=0$ e $V_{N+1}=0$ como indicado em Figura 4. O valor de $K_{h, j}$, para sistemas que operam a baixa pressão, pode ser estimado pela seguinte equação:

$K_{h, j}=\gamma_{h, j} \frac{P^{0}{ }_{h, j}}{P_{j}}$ sendo $\gamma_{h, j}$ o coeficiente de atividade do hexano na solução liquuida no estágio $j$ e $P_{h, j}^{o}$ a pressão de vapor do hexano puro no estágio $j$. Este coeficiente é obtido conforme PARAÍSO, ANDRADE \& ZEMP [8] e seu valor, considerado constante ao longo do equipamento, consta na Tabela 2, a seguir.

O valor desta pressão de vapor pode ser estimado pela equação de Antoine, conforme HIMMELBLAU [3],

$$
\ln \left(P_{h, j}^{0}\right)=15,8366-\frac{2697,55}{T_{j}-48,78}
$$

Sendo a pressão $P_{h, j}^{\circ}$ expressa em mmHg e $T_{j}$ em K.

Se as equações do balanço de massa forem agrupadas por componente, elas podem ser particionadas e colocadas como uma série de $C_{\mathrm{m}}$ matrizes tridiagonais onde a variável de saída para cada matriz equação é $x_{h^{\prime} j} \mathrm{em}$ todos os $N$ estágios do stripper.

As constantes $B_{j}$ e $C_{j}$ para cada componente depende somente das variáveis de iteração $T$ e $V$, uma vez que se considera que o valor da constante de equilíbrio, $K$, não varie significativamente com a composição ao longo da coluna.

A resolução da matriz tridiagonal gera os valores das composições do componente $i$ em cada estágio $j$, ou seja, os valores de $x_{h, j}$. Porém, os valores obtidos não são normalizados nesta etapa, mas utilizados diretamente para gerar novos valores de $L_{j}$ através da aplicação da seguinte equação

$L_{j}^{(k+1)}=L_{j}^{(k)}$

sendo que a equação (2.14) recebe a denominação de equação sum-rates e $k$ é o número de iterações realizadas.

Os valores de $L_{j}^{(k)}$ são obtidos a partir dos valores de $V_{j}^{(k)}$ calculados através da equação (2.6). Os correspondentes valores de $V_{j}^{(k+1)}$ são obtidos do balanço material global, o qual é derivado do somatório da equação (2.1) sobre os $c$ componentes, combinando o resultado com as equações (2.3) e (2.4) e somando aquele resultado sobre todos estágios $j$ até $N$ para se obter a seguinte equação:

$V_{j}=L_{j}-L_{N}+\sum_{m=j}^{N}\left(F_{m}+G_{m}\right)$

Os valores de $x_{i, j}$ necessitam ser normalizados uma vez que eles deverão ser utilizados posteriormente durante a iteração. Isto é feito através da seguinte equação:

$$
\left(x_{h, j}\right)_{\text {normalizado }}=\frac{x_{h, j}}{\sum_{i=1}^{c} x_{h, j}}
$$

Os correspondentes valores de $y_{h, j}$ são calculados através da equação (2.3) 
Neste ponto encerra-se o cálculo das composições de saída de cada estágio para o hexano tanto na fase líquida como na fase de vapor, $x_{h, j}$ e $y_{h, j}$ na iteração $k$, passando-se, então, para o cálculo do novos valores das temperaturas dos estágios, $T_{j}$.

Os valores de $T_{\mathrm{j}}$ são obtidos pela solução simultânea do conjunto de equações do balanço de energia para os $N$ estágios. As temperaturas estão embutidas nas correspondentes entalpias específicas das correspondentes correntes de líquido e vapor. Em geral, estas entalpias são não-lineares em relação à temperatura. Portanto, um procedimento de solução iterativa é necessário. Neste caso, o método de Newton-Raphson, segundo HENLEY \& SEADER [2], pode ser aplicado com sucesso.

A aplicação desse método para determinar os novos valores de $T_{j}$ é baseado na equação (2.2), cuja equação recursiva para o método de Newton-Raphson é a seguinte:

$\left(\frac{\partial H_{j}}{\partial T_{j-1}}\right)^{(k)} \Delta T_{j-1}{ }^{(k)}+\left(\frac{\partial H_{j}}{\partial T_{j}}\right)^{(k)} \Delta T_{j}{ }^{(k)}+\left(\frac{\partial H_{j}}{\partial T_{j+1}}\right)^{(k)} \Delta T_{j+1}{ }^{(k)}=-H_{j}{ }^{(k)}$

sendo:

$$
\begin{aligned}
& \Delta T_{j}{ }^{(k)}=T_{j}{ }^{(k+1)}-T_{j}{ }^{(k)} \\
& \frac{\partial H_{j}}{\partial T_{j-1}}=L_{j-1} \frac{\partial H^{L}{ }_{j-1}}{\partial T_{j-1}} \\
& \frac{\partial H_{j}}{\partial T_{j}}=-L_{j} \frac{\partial H_{j}^{L}}{\partial T_{j}}-V_{j} \frac{\partial H^{V}}{\partial T_{j}} \\
& \frac{\partial H_{j}}{\partial T_{j+1}}=V_{j+1} \frac{\partial H^{V}{ }_{j+1}}{\partial T_{j+1}}
\end{aligned}
$$

As derivadas parciais dependem das correlações de entalpia que são utilizadas. Neste trabalho considerase que a entalpia é independente da composição, portanto, usar-se-á para os cálculos de $H_{j}^{L}$ de $H_{j}^{V}$ e suas respectivas derivadas, as seguintes equações:

$H_{j}^{L}=x_{h, j} c_{p h}\left(T_{j}-T_{r}\right)+\left(1-x_{h, j}\right) c_{p o}\left(T_{j-} T_{r}\right)$

sendo $c_{p h}$ e $c_{p o}$ os calores específicos do hexano líquido e do óleo de soja, respectivamente, e $T_{r}$ a temperatura de referência.

$H_{j}^{V}=y_{h, j}\left[\tau_{h}+c_{p h y}\left(T_{j}-T_{r}\right)\right]+\left(1-y_{h, j}\right)\left[\tau_{h}+c_{p y a}\left(T_{j}-T_{r}\right)\right]$

sendo $\tau_{h}$ o calor latente de vaporização do hexano na temperatura de referência $T_{r}$, e $c_{p h y}$ e $c_{p y a}$ os calores específicos do hexano e da água no estado de vapor.

As $N$ equações formadas a partir da equação (2.17) formam uma equação matriz tridiagonal que é linear em relação a $\Delta T_{j}$. A solução da equação matriz gera o conjunto de correções $\Delta T_{j}^{(k)}$. Novos supostos valores de $T_{j}$ são determinados da seguinte forma:

$T_{j}^{(k+1)}=T_{j}^{(k)}+f \Delta T_{j}^{(k)}$

sendo $f$ um fator escalar de atenuação que é útil quando os valores supostos inicialmente não estão razoavelmente próximos dos valores verdadeiros. Geralmente, $f$ é tomado como unitário. Quando todas correções $\Delta T_{j}^{(k)}$ estão próximas de zero, deve-se procurar um critério para determinar se a convergência foi alcançada. Neste caso, uma maneira de verificar tal convergência é através do seguinte critério:

$\tau=\sum_{j=1}^{N}\left[T_{j}^{(k)}-T_{j}^{(k-1)}\right]^{2} \leq 0,01 N$

Substituição sucessiva é geralmente usada no processo de iteração, ou seja, valores de $V_{j}$ e de $T_{j}$ gerados a partir das equações (2.15) e (2.24), respectivamente, durante uma iteração são usados diretamente para iniciar a próxima iteração.

\section{3 - Simulação da operação de stripping}

\subsection{1 - Desenvolvimento do algoritmo}

Aqui, apresentam-se os procedimentos adequados para análise de desempenho da operação de stripping da miscela.

Como já foi abordado anteriormente, fixando-se todas as vazões de alimentação $F_{j}$ ao lado de sua concentração $z_{h, j}$ e condições termodinâmicas de pressão $P^{F}{ }_{j}$ e temperatura $T^{F}$, as vazões de vapor direto $G_{j}$ e suas condições termodinâmicas $T^{G} j$ e $P_{j}^{G}$, a pressão de estágio $P_{j}$ e o número de estágios $N$; determina-se os valores de $x_{h, j}, y_{h, j}, L_{j}, V_{j}$ e $T_{\mathrm{j}}$. Para tanto, as seguintes etapas são necessárias: I) supõe-se um valor inicial para $T_{j}$ e $V_{j}$; II) calcula-se a pressão de vapor do hexano e a constante de equilíbrio; III) calcula-se os valores das concentrações do hexano, $x_{h, j}$, resolvendo-se equação matriz (2.7); IV) calcula-se novos valores de $L_{j}$ a partir da equação sum-rates (2.14) e dos novos valores de $V_{j}$ utilizando a equação (2.15); V) normaliza-se os valores de $x$ para cada estágio através da equação (2.16) e calcula-se os valores de $y$ correspondentes; VII) calculase os novos valores de $T_{j}$ a partir da equação (2.2) em conjunto com as equações do método de NewtonRaphson; e, VIII) se necessário, faz-se a iteração, conforme mostra a Figura 5.

O programa computacional derivado do algoritmo da Figura 5 é composto de três elementos: entrada de dados, programa principal e saída de dados.

A entrada de dados se compõe basicamente do coeficiente de atividade do hexano na solução e daqueles valores considerados fixos na condição de funcionamento do stripper. Esses dados são: vazão de alimentação do estágio, $F_{j}$ em moles/hora; fração molar do hexano na alimentação, $z_{h, j}$ em moles de hexano/moles de miscela; e, pressão do estágio, $P_{j}$ em mmHg. 


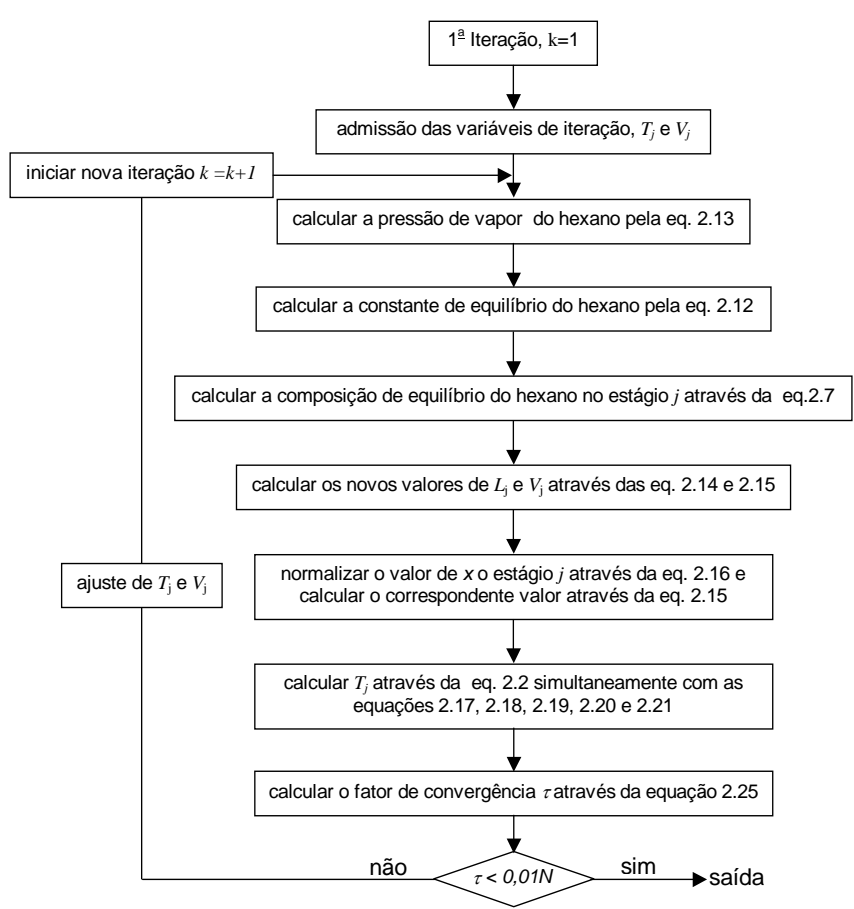

FIGURA 5. Estrutura do algoritmo para a simulação do stripper

O programa principal é o elemento que realiza seqüencialmente os cálculos e as iterações necessárias.

A saída de dados compõe-se dos seguintes dados: as composições molares do hexano no topo e no fundo do stripper, $y_{h}$ e $x_{h}$ respectivamente, em moles de hexano/moles de miscela; a temperatura de cada estágio, $T_{j}$ em ${ }^{\circ} \mathrm{C}$; e, a vazão de vapor, $V_{j}$ em moles/hora.

\subsection{2 - Dados operacionais do stripper}

A fim de cumprir o objetivo proposto inicialmente nesta seção, fez-se um levantamento de dados operacionais na indústria da COAMO. Utilizando-se ora somente os dados operacionais ora em conjunto com os dados simulados, em diferentes situações, foi possivel obter vários resultados que podem servir de base para um programa de redução de custos numa indústria de obtenção de óleo de soja. O stripper da COAMO apresenta uma configuração geral semelhante à da Figura 6 mostrada a seguir.

Conforme mostra a Figura 6, o stripper da COAMO é composto de dois estágios e opera sob pressão na faixa de 450 a 600 mmHg. Este equipamento recebe a miscela, que vem do sistema de evaporação com uma concentração, em termos de fração mássica, em torno de $98 \%$ de óleo e temperatura em torno de $120^{\circ} \mathrm{C}$, com a função de tornar o óleo de soja praticamente livre de hexano. A miscela entra no seu topo de forma pulverizada e flui para baixo entrando em contato direto com o vapor d'água. Este contato possibilita a desorção do hexano, de tal forma, que o óleo sai no fundo com uma concentração mássica de hexano em torno de 1300ppm. $\mathrm{O}$ vapor d'água entra, em cada estágio, superaquecido a uma pressão e a uma temperatura de aproximadamente $4,0 \mathrm{kgf} / \mathrm{cm}^{2}$ e de $180^{\circ} \mathrm{C}$, respectivamente.

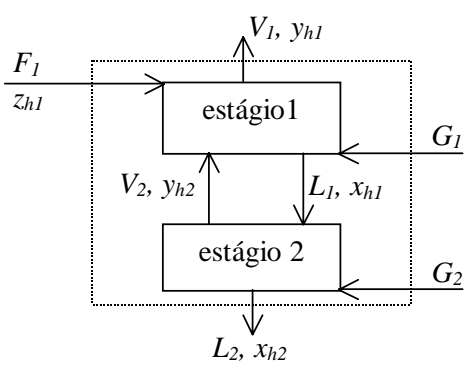

FIGURA 6. Esquema geral do stripper da COAMO

É importante também ressaltar, como já foi mostrado acima, que o stripper da fábrica da COAMO possui dois estágios cada qual com sua entrada de vapor d'água. No entanto, na atualidade a entrada de vapor está sendo feita apenas no primeiro estágio. O argumento dos operadores da fábrica, para tal, é que a injeção de vapor no segundo estágio possibilita a saída de água juntamente com o óleo no fundo do extrator; ainda segundo os operadores isto deve ser evitado uma vez que irá prejudicar o desempenho da operação seguinte, denominada de secagem de óleo. Entretanto, a não injeção de vapor nesse segundo estágio aumenta significativamente o consumo de vapor e diminui a recuperação do solvente como será mostrado, a seguir, através dos resultados simulados.

A Tabela 2 apresenta os operacionais obtidos na indústria da cooperativa COAMO, os quais serão comparados com os resultados obtidos através do processo de simulação a ser realizado.

TABELA 2. Dados operacionais do stripper da COAMO

\begin{tabular}{lcc}
\hline \multicolumn{1}{c}{ Dados operacionais } & símbolos & valores \\
\hline vazão da miscela na entrada do stripper & $F_{1}$ & $7490,0 \mathrm{~kg} / \mathrm{h}$ \\
fração mássica do hexano na entrada do stripper & $Z_{h}$ & $2,0 \%$ \\
temperatura da miscela na entrada & $T_{1}$ & $119,7^{\circ} \mathrm{C}$ \\
pressão no $1^{\circ}$ estágio & $P_{1}$ & $470 \mathrm{mmHg}$ \\
pressão no $2^{\circ}$ estágio & $P_{2}$ & $560 \mathrm{mmHg}$ \\
vazão mássica do óleo bruto no fundo do stripper & $L_{2}$ & $7.345,0 \mathrm{~kg} / \mathrm{h}$ \\
fração mássica do hexano no fundo do stripper & $X_{h 2}$ & $1360 \mathrm{ppm}$ \\
temperatura da miscela no fundo do stripper & $T_{2}$ & $114{ }^{\circ} \mathrm{C}$ \\
temperatura do vapor direto & $T^{G}$ & $180^{\circ} \mathrm{C}$ \\
pressão do vapor direto & $P^{G}$ & $4,0 \mathrm{kgf} / \mathrm{cm}^{2}$ \\
coeficiente de atividade & $m_{, j}$ & 1,24 \\
\hline
\end{tabular}

Os dados de vazão e de concentração presentes na Tabela 2 coletados na COAMO estão expressos em unidades de massa. Para transformá-los em unidades molares utiliza-se o peso molecular do hexano puro, que é igual a $86,17 \mathrm{~g} / \mathrm{mol}$ e o peso molecular do óleo como sendo a média dos pesos moleculares dos ácidos graxos presentes no óleo, ou seja, 795,6g/mol.

\section{3 - RESULTADOS E DISCUSSÃO}

Os resultados obtidos com as simulações do stripper serão apresentados de duas maneiras. A primeira de- 
las se apresenta como a mais relevante: a comparação entre os valores de saída das vazões, das concentrações e das temperaturas obtidos através do modelo e aqueles coletados na indústria da cooperativa COAMO. A segunda maneira, se refere à verificação do comportamento de determinadas variáveis provenientes de alterações impostas nas condições de operação do processo.

\section{1 - Comparação dos resultados do modelo com os resultados operacionais do stripper.}

Os resultados obtidos através do modelo são comparados com os dados operacionais obtidos na indústria. A fim de realizar esta análise da forma mais fiel possivel, a simulação foi realizada introduzindo vapor direto apenas no primeiro estágio e utilizando-se os dados de entrada conforme é especificado no algoritmo da Figura 5. A Tabela 3 mostra esses dados.

TABELA 3. Resultados obtidos com o modelo e suas comparações com os dados reais

\begin{tabular}{lccc}
\hline \multicolumn{1}{c}{ Resultados obtidos } & símbolo & valor real & valor simulado \\
\hline vazão mássica de vapor no topo & $V_{1}$ & - & $1100,0 \mathrm{~kg} / \mathrm{h}$ \\
vazão mássica de líquido no fundo & $L_{2}$ & $7345,0 \mathrm{~kg} / \mathrm{h}$ & $7358,0 \mathrm{~kg} / \mathrm{h}$ \\
fração mássica do hexano no líquido do fundo & $X_{h 2}$ & $1360 \mathrm{ppm}$ & $1413 \mathrm{ppm}$ \\
fração mássica do hexano no vapor do topo & $Y_{h 1}$ & - & 0,126 \\
temperatura do líquido na saída do stripper & $T_{2}$ & $114,0^{\circ} \mathrm{C}$ & $113,6^{\circ} \mathrm{C}$ \\
consumo de vapor d'água & $G_{1}$ & - & $960,0 \mathrm{~kg} / \mathrm{h}$ \\
\hline
\end{tabular}

A Tabela 3 mostra que embora, a COAMO não efetua medidas da vazão de vapores que sai no topo do stripper e, nem do consumo de vapor d'água direto utilizado, a empresa avalia o desempenho de seu processo somente através da vazão mássica de óleo bruto, do teor de hexano que sai junto com o óleo e da temperatura do óleo, que saem no fundo do stripper. Assim, somente estes puderam ser comparados diretamente com aqueles obtidos pelo modelo. A comparação mostra uma boa aproximação entre os dados que foram coletados e os valores obtidos através do modelo aplicado. Pode-se observar que o valor da fração mássica do hexano é de 0,00136 ou 1360ppm na indústria e de 0,001413 ou 1413 ppm através do modelo, ou seja, a diferença entre o valor real e o valor simulado apresenta um erro relativo de apenas 3,9\%; além disso, esses valores estão de acordo com os padrões aceitos para o hexano residual, conforme mostra a Tabela 2. Com relação à temperatura do óleo na saída, pode-se observar que o seu valor real na indústria é de $114,0^{\circ} \mathrm{C}$ na indústria, e de $113,6^{\circ} \mathrm{C}$ através da simulação, portanto, com um erro de apenas $0,4 \%$. Com relação ao dado de consumo de vapor d'água direto no stripper, apesar de não existir o dado real da indústria, alguns comentários podem ser ditos. O consumo obtido através do modelo foi de $24 \mathrm{~kg}$ de vapor por tonelada de soja esmagada; este valor é $60 \%$ do valor estimado por JONGENEELEN [5] é de 40kg de vapor por tonelada de soja processada em todas opearações de Destilação da Miscela. Isto indica que a COAMO está gastando mais vapor que o recomendado, uma vez que a operação de evaporação, que também compõe a destilação da miscela, consome $34 \mathrm{~kg}$ de vapor por tonelada de soja esmagada, conforme PARAÍSO [7]. Cabe ressaltar, ainda que a simulação foi efetuada conforme está operando atualmente a coluna da indústria da COAMO com a introdução de vapor somente no primeiro estágio; como será visto posteriormente, no tópico 4.3, esta forma de operar traz ineficiência para o sistema tanto na recuperação de hexano como no consumo de vapor.

\section{2 - Influência da concentração da alimentação nos resultados operacionais do stripper}

O objetivo principal aqui é mostrar o efeito que a concentração de hexano na entrada afeta os resultados de saída do equipamento. A fim de realizar esta análise utilizou-se os dados da Tabela 2 e aplicou-se o modelo para dois valores de concentração, em massa: $2 \%$ e $1,2 \%$. Os resultados obtidos são mostrados na Tabela 4 .

TABELA 4. Comparação dos resultados obtidos para concentrações diferentes

\begin{tabular}{lccc}
\hline \multicolumn{4}{c}{ Resultados obtidos } \\
\hline Valores de saída no stripper & símbolo & $\begin{array}{c}\text { valor obtido } \\
\left(Z_{h}=2,0 \%\right)\end{array}$ & $\begin{array}{c}\text { valor obtido } \\
\left(Z_{h}=1,2 \%\right)\end{array}$ \\
\hline vazão mássica de vapor no topo & $V_{1}$ & $1100 \mathrm{~kg} / \mathrm{h}$ & $601 \mathrm{~kg} / \mathrm{h}$ \\
vazão mássica de líquido no fundo & $L_{2}$ & $7358 \mathrm{~kg} / \mathrm{h}$ & $7346 \mathrm{~kg} / \mathrm{h}$ \\
fração mássica do hexano do fundo & $X_{h 2}$ & $1413 \mathrm{ppm}$ & $1394 \mathrm{ppm}$ \\
fração mássica do hexano no topo & $Y_{h 1}$ & 0,1260 & 0,1312 \\
temperatura do líquido no fundo & $T_{2}$ & $113,6^{\circ} \mathrm{C}$ & $115,7^{\circ} \mathrm{C}$ \\
consumo de vapor d'água direto & $G_{1}$ & $960,0 \mathrm{~kg} / \mathrm{h}$ & $522,0 \mathrm{~kg} / \mathrm{h}$ \\
\hline
\end{tabular}

A Tabela 4 mostra que a redução na concentração do hexano na alimentação provoca os seguintes efeitos relevantes nos dados de saida do stripper:

- uma redução significativa do consumo de vapor direto na operação com a redução da concentração do hexano na alimentação passando de $960 \mathrm{~kg} / \mathrm{h}$ para $522 \mathrm{~kg} / \mathrm{h}$, e mesmo com esta redução, a quantidade de hexano residual no óleo, nos dois casos, se manteve próximos, em torno de 1400ppm;

- as vazões mássicas no topo do stripper diminuiu, passando de $1100 \mathrm{~kg} / \mathrm{h}$ para $601 \mathrm{~kg} / \mathrm{h}$, assim como a vazão no fundo que passou de $7358 \mathrm{~kg} / \mathrm{h}$ para $7346 \mathrm{~kg} / \mathrm{h}$. Estas reduções se explicam devido à menor quantidade de hexano no sistema, bem como o menor consumo de vapor direto; e

- a temperatura no stripper aumentou, passando de $113,6^{\circ} \mathrm{C}$ para $115,7^{\circ} \mathrm{C}$. Este aumento pode ser justificado, uma vez que a quantidade de hexano evaporada no segundo caso foi inferior ao primeiro caso, com isso o resfriamento do sistema foi menor. 


\section{3 - Efeito do modo de injeção de vapor direto nos resultados operacionais do stripper}

Aqui, pretende-se verificar os efeitos produzidos nos resultados operacionais desse equipamento quando se introduz o vapor direto nos dois estágios. Para tal, introduz-se vapor direto nos dois estágios sendo que as vazões $G_{1}$ e $G_{2}$ variam cada uma de 0 a $960,0 \mathrm{~kg} / \mathrm{h}$, os demais dados solicitados pelo modelo são como os da Tabela 2. Os resultados obtidos através das simulações são mostrados na Tabela 5.

TABELA 5. Influência da injeção de vapor nos resultados operacionais do stripper

\begin{tabular}{ccccccc}
\hline \multicolumn{6}{c}{ Resultados obtidos } \\
\hline $\begin{array}{c}\mathrm{G}_{1} \\
(\mathrm{~kg} / \mathrm{h})\end{array}$ & $\begin{array}{c}\mathrm{G}_{2} \\
(\mathrm{~kg} / \mathrm{h})\end{array}$ & $\begin{array}{c}L_{2} \\
(\mathrm{~kg} / \mathrm{h})\end{array}$ & $\begin{array}{c}X_{\mathrm{h} 2} \\
(\mathrm{ppm})\end{array}$ & $\begin{array}{c}T_{1} \\
\left({ }^{\circ} \mathrm{C}\right)\end{array}$ & $\begin{array}{c}T_{2} \\
\left({ }^{\circ} \mathrm{C}\right)\end{array}$ & $\begin{array}{c}\text { hexano não recupe } \\
(\mathrm{kg} / \mathrm{h})\end{array}$ \\
\hline 960,0 & 0,0 & 7358,0 & 1413 & 113,5 & 113,5 & 10,4 \\
780,0 & 180,0 & 7350,4 & 479 & 114,0 & 113,2 & 3,5 \\
720,0 & 240,0 & 7349,8 & 393 & 114,1 & 113,2 & 2,9 \\
670,0 & 290,0 & 7349,4 & 342 & 114,2 & 113,1 & 2,5 \\
580,0 & 380,0 & 7348,9 & 276 & 114,5 & 113,1 & 2,0 \\
220,0 & 740,0 & 7348,0 & 156 & 115,4 & 113,0 & 1,2 \\
0,0 & 960,0 & 7347,7 & 120 & 115,9 & 112,9 & 0,9 \\
\hline
\end{tabular}

Uma análise dos resultados apresentados na Tabela 5 mostra que a melhor condição operacional do stripper é a introdução integral do vapor necessário apenas no último estágio desse equipamento. A recuperação do hexano foi superior a todas as outras situações simuladas, especialmente àquela em que se introduz o vapor apenas no primeiro estágio. Além disso, a temperatura no segundo estágio manteve-se um valor próximo ao valor real de $113^{\circ} \mathrm{C}$, e até apresentou um certo declínio para o valor de $112,9^{\circ} \mathrm{C}$, o que é benéfico para a qualidade do óleo produzido.

\section{4 - CONCLUSÕES}

O objetivo deste trabalho foi apresentar um modelo capaz de analisar as condições de operação stripping na tarefa de recuperação do hexano ainda presente no óleo de soja após a evaporação.

Os resultados obtidos mostraram que o modelo proposto é bastante coerente e pode ser perfeitamente utilizado como ferramenta no processo de análise e otimização da operação de stripping do hexano presente no óleo.

\section{5 - REFERÊNCIAS BIBLIOGRÁFICAS}

[1] BARBOSA, M.Z.; FREITAS, S.M. de; FRANCA, T.J.F. Considerações sobre os desafios da cadeia de produção de óleo de soja no Brasil, óleos \& Grãos, p. 44, Setembro/Outubro, 1998.

[2] HENLEY, J.E.; SEADER, D.J. Equilibrium-Stage Separation Operations in Chemical Engineering, John Wiley \& Sons, New York, 1981.

[3] HIMMELBLAU, D.M. Engenharia Química: Princípios e Cálculos. Trad. Jossyl de Souza Peixoto, Sindicato Nacional dos Editores de Livros, Rio de Janeiro, 1984.

[4] HOLLAND, C.D. Fundamentals and Modeling of Separation Processes, Prentice-Hall, New Jersey, 1975.

[5] JONGENEELEN, H.P.J. Energy Conservation in Solvent Extration Plants, JAOCS Journal of the American Oil Chemists Society, v. 53, p. 291, June, 1976.

[6] KEMPER, G.T. Minimização da perda de solvente, Grãos \& Óleos, p. 22, Setembro/Outubro, 1998.

[7] PARAÍSO, P.R. Modelagem e Análise do Processo de Obtenção do Óleo de Soja, 2001, Campinas. Tese de Doutorado - FEQ/UNICAMP, 212 p.

[8] PARAÍSO, P.R.; ANDRADE, C.M.G.; ZEMP, R.J. Destilação da Miscela I: Modelagem e Simulação da Evaporação do Hexano. Ciênc. Tecnol. Aliment., v. 23, n. 3, p. 459-467, set.-dez., 2003.

[9] SUJATA, A.D. Fundamentals and Modeling of Separation Processes in: HOLLAND, C. D. Prentice-Hall, New Jersey, 1975.

\section{6 - AGRADECIMENTOS}

À COAMO, que disponibilizou os dados operacionais, situada no município de Campo Mourão-PR. 